\title{
New Result of Analytic Functions Related to Hurwitz Zeta Function
}

\author{
F. Ghanim ${ }^{1}$ and M. Darus ${ }^{2}$ \\ ${ }^{1}$ Department of Mathematics, College of Sciences, University of Sharjah, Sharjah, United Arab Emirates \\ ${ }^{2}$ School of Mathematical Sciences, Faculty of Science and Technology, Universiti Kebangsaan Malaysia, \\ 43600 Bangi, Selangor Darul Ehsan, Malaysia
}

Correspondence should be addressed to F. Ghanim; firas.zangnaa@gmail.com

Received 25 September 2013; Accepted 11 November 2013

Academic Editors: L. Gosse, T. Li, and P. Wang

Copyright (c) 2013 F. Ghanim and M. Darus. This is an open access article distributed under the Creative Commons Attribution License, which permits unrestricted use, distribution, and reproduction in any medium, provided the original work is properly cited.

By using a linear operator, we obtain some new results for a normalized analytic function $f$ defined by means of the Hadamard product of Hurwitz zeta function. A class related to this function will be introduced and the properties will be discussed.

\section{Introduction}

A meromorphic function is a single-valued function, that is, analytic in all but possibly a discrete subset of its domain, and at those singularities it must go to infinity like a polynomial (i.e., these exceptional points must be poles and not essential singularities). A simpler definition states that a meromorphic function $f(z)$ is a function of the form

$$
f(z)=\frac{g(z)}{h(z)},
$$

where $g(z)$ and $h(z)$ are entire functions with $h(z) \neq 0$ (see [1, page 64]). A meromorphic function therefore may only have finite-order, isolated poles and zeros and no essential singularities in its domain. A meromorphic function with an infinite number of poles is exemplified by $\csc (1 / z)$ on the punctured disk $U^{*}=\{z: 0<|z|<1\}$.

An equivalent definition of a meromorphic function is a complex analytic map to the Riemann sphere. For example, the Gamma function is meromorphic in the whole complex plane; see $[1,2]$.

In the present paper, we will derive some properties of univalent functions defined by means of the Hadamard product of Hurwitz Zeta function; a class related to this function will be introduced and the properties of the liner operator $L_{a}^{t}(\alpha, \beta) f(z)$ will be discussed.

\section{Preliminaries}

Let $\Sigma$ denote the class of meromorphic functions $f(z)$ normalized by

$$
f(z)=\frac{1}{z}+\sum_{n=1}^{\infty} a_{n} z^{n},
$$

which are analytic in the punctured unit disk $U^{*}$. For $0 \leq \beta$, we denote by $S^{*}(\beta)$ and $k(\beta)$ the subclasses of $\Sigma$ consisting of all meromorphic functions which are, respectively, starlike of order $\beta$ and convex of order $\beta$ in $U^{*}$.

For functions $f_{j}(z)(j=1 ; 2)$ defined by

$$
f_{j}(z)=\frac{1}{z}+\sum_{n=1}^{\infty} a_{n, j} z^{n},
$$

we denote the Hadamard product (or convolution) of $f_{1}(z)$ and $f_{2}(z)$ by

$$
\left(f_{1} * f_{2}\right)=\frac{1}{z}+\sum_{n=1}^{\infty} a_{n, 1} a_{n, 2} z^{n} .
$$

Let us define the function $\widetilde{\phi}(\alpha, \beta ; z)$ by

$$
\tilde{\phi}(\alpha, \beta ; z)=\frac{1}{z}+\sum_{n=0}^{\infty} \frac{(\alpha)_{n+1}}{(\beta)_{n+1}} z^{n},
$$


for $\beta \neq 0,-1,-2, \ldots$, and $\alpha \in \mathbb{C} /\{0\}$, where $(\lambda) n=\lambda(\lambda+1)_{n+1}$ is the Pochhammer symbol. We note that

$$
\widetilde{\phi}(\alpha, \beta ; z)=\frac{1}{z^{2}} F_{1}(1, \alpha, \beta ; z),
$$

where

$$
{ }_{2} F_{1}(b, \alpha, \beta ; z)=\sum_{n=0}^{\infty} \frac{(b)_{n}(\alpha)_{n}}{(\beta)_{n}} \frac{z^{n}}{n !}
$$

is the well-known Gaussian hypergeometric function.

We recall here a general Hurwitz-Lerch-Zeta function, which is defined in $[3,4]$ by the following series:

$$
\Phi(z, t, a)=\frac{1}{a^{t}}+\sum_{n=1}^{\infty} \frac{z^{n}}{(n+a)^{t}}
$$

$\left(a \in \mathbb{C} / \mathbb{Z}_{0}^{-}, \mathbb{Z}_{0}^{-}=\{0,-1,-2, \ldots\} ; t \in \mathbb{C}\right.$ when $z \in U=U^{*} \subset$ $\{0\} ; \mathfrak{R}(t)>1$ when $z \in \partial U)$.

Important special cases of the function $\Phi(z, t, a)$ include, for example, the Riemann zeta function $\zeta(t)=\Phi(1, t, 1)$, the Hurwitz zeta function $\zeta(t, a)=\Phi(1, t, a)$, the Lerch zeta function $l_{t}(\zeta)=\Phi\left(\exp ^{2 \pi i \xi}, t, 1\right),(\xi \in \mathbb{R}, \mathfrak{R}(t)>1)$, and the polylogarithm $L_{t}^{i}(z)=z \Phi(z, t, a)$. Recent results on $\Phi(z, t, a)$ can be found in the expositions $[5,6]$. By making use of the following normalized function we define

$$
\begin{aligned}
G_{t, a}(z) & =(1+a)^{t}\left[\Phi(z, t, a)-a^{t}+\frac{1}{z(1+a)^{t}}\right] \\
& =\frac{1}{z}+\sum_{n=1}^{\infty}\left(\frac{1+a}{n+a}\right)^{t} z^{n}, \quad\left(z \in U^{*}\right) .
\end{aligned}
$$

Corresponding to the functions $G_{t, a}(z)$ and using the Hadamard product for $f(z) \in \Sigma$, we define a new linear operator $L_{t, a}(\alpha, \beta)$ on $\Sigma$ by the following series:

$$
\begin{aligned}
& L_{a}^{t}(\alpha, \beta) f(z)= \phi(\alpha, \beta ; z) * G_{t, a}(z) \\
&=\frac{1}{z}+\sum_{n=1}^{\infty} \frac{(\alpha)_{n+1}}{(\beta)_{n+1}}\left(\frac{1+a}{n+a}\right)^{t} a_{n} z^{n} . \\
& \quad\left(z \in U^{*}\right) .
\end{aligned}
$$

The meromorphic functions with the generalized hypergeometric functions were considered recently by many others; see, for example, [7-12].

It follows from (10) that

$$
\begin{aligned}
& z\left(L_{a}^{t}(\alpha, \beta) f(z)\right)^{\prime} \\
& \quad=\alpha\left(L_{a}^{t}(\alpha+1, \beta) f(z)\right)-(\alpha+1) L_{a}^{t}(\alpha, \beta) f(z) .
\end{aligned}
$$

In order to prove our main results, we recall the following lemma according to Yang [13].

Lemma 1. Let $q(z)=1+q_{n} z^{n}+q_{n+1} z^{n+1}+\cdots$ be analytic functions in $U=U^{*} \cup\{0\}$ with $q(z) \neq 0$ for $z \in U$. If

$$
\mathfrak{R}\left\{1+a \frac{z q^{\prime}(z)}{q^{2}(z)}\right\}<M, \quad(z \in U),
$$

where $a>0$, and

$$
1<M \leq \frac{n a}{2 \log 2}
$$

then

$$
\Re\left\{\frac{1}{q(z)}\right\}>1-\frac{2(M-1)}{n a} \log 2, \quad(z \in U) .
$$

The bound in (14) is the best possible.

\section{Main Results}

We begin with the following theorem.

Theorem 2. Let $\alpha+1>0, L_{a}^{t}(\alpha, \beta) f(z) / L_{a}^{t}(\alpha+1, \beta) f(z) \neq 0$ for $z \in U^{*}$ and suppose that

$$
\begin{gathered}
\Re\left\{1+\frac{L_{a}^{t}(\alpha+1, \beta) f(z)}{(\alpha+1) L_{a}^{t}(\alpha, \beta) f(z)}\left(1+\frac{\alpha L_{a}^{t}(\alpha+1, \beta) f(z)}{L_{a}^{t}(\alpha, \beta) f(z)}\right)\right. \\
\left.-\frac{L_{a}^{t}(\alpha+2, \beta) f(z)}{L_{a}^{t}(\alpha, \beta) f(z)}\right\}<M,
\end{gathered}
$$

where

$$
1<M \leq \frac{n}{2(\alpha+1) \log 2} .
$$

Then

$$
\begin{aligned}
& \Re\left\{\frac{L_{a}^{t}(\alpha+1, \beta) f(z)}{L_{a}^{t}(\alpha, \beta) f(z)}\right\} \\
& \quad>1-\frac{2(\alpha+1)(M-1)}{n} \log 2, \quad\left(z \in U^{*}\right) .
\end{aligned}
$$

The bound in (17) is the best possible.

Proof. Define the function $q(z)$ by

$$
q(z)=\frac{L_{a}^{t}(\alpha, \beta) f(z)}{L_{a}^{t}(\alpha+1, \beta) f(z)} .
$$

Then, clearly $q(z)=1+q_{n} z^{n}+q_{n+1} z^{n+1}+\cdots$ analytic function in $U^{*}$ with $q(z) \neq 0$ for $z \in U^{*}$. It follows from (18) and (11) that

$$
\frac{z q^{\prime}(z)}{q(z)}=\frac{z\left(L_{a}^{t}(\alpha, \beta) f(z)\right)^{\prime}}{L_{a}^{t}(\alpha, \beta) f(z)}-\frac{z\left(L_{a}^{t}(\alpha+1, \beta) f(z)\right)^{\prime}}{L_{p}^{*}(a+1, c) f(z)}
$$

by making use of the familiar identity (11) in (19), we obtain

$$
\frac{L_{a}^{t}(\alpha+2, \beta) f(z)}{L_{a}^{t}(\alpha+1, \beta) f(z)}=\frac{1}{\alpha+1}+\frac{1}{(\alpha+1) q(z)}-\frac{z q^{\prime}(z)}{(\alpha+1) q(z)}
$$


or, equivalent,

$$
\begin{aligned}
1+ & \frac{1}{(\alpha+1)} \frac{z q^{\prime}(z)}{q^{2}(z)} \\
= & 1+\frac{L_{a}^{t}(\alpha+1, \beta) f(z)}{(\alpha+1) L_{a}^{t}(\alpha, \beta) f(z)}\left(1+\frac{\alpha L_{a}^{t}(\alpha+1, \beta) f(z)}{L_{a}^{t}(\alpha, \beta) f(z)}\right) \\
& -\frac{L_{a}^{t}(\alpha+2, \beta) f(z)}{L_{a}^{t}(\alpha+1, \beta) f(z)} .
\end{aligned}
$$

Applying Lemma 1 , with $a=1 /(1+\alpha)$, we get the required result.

Letting $\alpha=\beta=1$ in Theorem 2, we have the following.

Corollary 3. Let $G_{t, a}(z) / z\left(G_{t, a}(z)\right)^{\prime} \neq 0$ for $z \in U^{*}$ and suppose that

$$
\begin{aligned}
\Re\{ & 1+\frac{z\left(G_{t, a}(z)\right)^{\prime}}{2 G_{t, a}(z)}\left(1+\frac{z\left(G_{t, a}(z)\right)^{\prime}}{G_{t, a}(z)}\right) \\
& \left.-\frac{z\left(G_{t, a}(z)\right)^{\prime}+(1 / 2)\left(G_{t, a}(z)\right)^{\prime \prime}}{G_{t, a}(z)}\right\}<M,
\end{aligned}
$$

where

$$
1<M \leq \frac{n}{4 \log 2}
$$

Then

$$
\mathfrak{R}\left\{\frac{z\left(G_{t, a}(z)\right)^{\prime}}{G_{t, a}(z)}\right\}>1-\frac{4(M-1)}{n} \log 2, \quad\left(z \in U^{*}\right) .
$$

The bound in (24) is the best possible.

Letting $M=1+n / 4 \log 2$ in Corollary 3 , we have the following.

Corollary 4. Let $G_{t, a}(z) / z\left(G_{t, a}(z)\right)^{\prime} \neq 0$ and $t=0$ for $z \in U^{*}$ and suppose that

$$
\begin{aligned}
& \Re\left\{1+\frac{z f^{\prime}(z)}{2 f(z)}\left(1+\frac{z f^{\prime}(z)}{f(z)}\right)-\frac{z f^{\prime}(z)+(1 / 2) z^{2} f^{\prime \prime}(z)}{f(z)}\right\} \\
& \quad<1+\frac{n}{4 \log 2} .
\end{aligned}
$$

Then $f(z)$ is starlike in $U^{*}$.

Theorem 5. Let $\delta(\alpha+1)>0, z L_{a}^{t}(\alpha+1, \beta) f(z) \neq 0$ for $z \in U^{*}$ and suppose that

$$
\mathfrak{R}\left\{\left(z L_{a}^{t}(\alpha+1, \beta) f(z)\right)^{\delta}\left(\frac{L_{a}^{t}(\alpha+2, \beta) f(z)}{L_{a}^{t}(\alpha+1, \beta) f(z)}\right)\right\}<M,
$$

where

$$
1<M \leq \frac{n}{2 \delta(\alpha+1) \log 2}
$$

Then

$$
\begin{aligned}
& \mathfrak{R}\left(z L_{a}^{t}(\alpha+1, \beta) f(z)\right)^{\delta} \\
& \quad>1-\frac{2 \delta(\alpha+1)(M-1)}{n} \log 2, \quad\left(z \in U^{*}\right) .
\end{aligned}
$$

The bound in (28) is the best possible.

Proof. Define the function $q(z)$ by

$$
q(z)=\left(z L_{a}^{t}(\alpha+1, \beta) f(z)\right)^{\delta}
$$

Then, clearly $q(z)=1+q_{n} z^{n}+q_{n+1} z^{n+1}+\cdots$ analytic function in $U^{*}$ with $q(z) \neq 0$ for $z \in U^{*}$. It follows from (29) that

$$
\frac{z q^{\prime}(z)}{\delta q(z)}=\frac{z\left(L_{a}^{t}(\alpha+1, \beta) f(z)\right)^{\prime}}{L_{a}^{t}(\alpha+1, \beta) f(z)}-1
$$

by making use of the familiar identity (11) in (30), we get

$$
\frac{L_{a}^{t}(\alpha+2, \beta) f(z)}{L_{a}^{t}(\alpha+1, \beta) f(z)}-1=\frac{1}{\delta(\alpha+1)} \frac{z q^{\prime}(z)}{q(z)},
$$

or, equivalent

$$
\begin{aligned}
1 & +\frac{1}{\delta(\alpha+1)} \frac{z q^{\prime}(z)}{q^{2}(z)} \\
& =\left(z L_{a}^{t}(\alpha+1, \beta) f(z)\right)^{\delta}\left(\frac{L_{a}^{t}(\alpha+2, \beta) f(z)}{L_{a}^{t}(\alpha+1, \beta) f(z)}\right) .
\end{aligned}
$$

Applying Lemma 1 , with $a=1 /(1+\alpha)$, we get the required result.

Letting $\alpha=\beta=1$ in Theorem 5 , we have

Corollary 6. Let $\delta>0, G_{t, a}(z) \neq 0$ for $z \in U^{*}$ and suppose that

$$
\Re\left\{\left(z G_{t, a}(z)\right)^{\delta}\left(\frac{z\left(G_{t, a}(z)\right)^{\prime}+(1 / 2)\left(G_{t, a}(z)\right)^{\prime \prime}}{G_{t, a}(z)}\right)\right\}<M,
$$

where

$$
1<M \leq \frac{n}{4 \delta \log 2}
$$

Then

$$
\mathfrak{R}\left(z G_{t, a}(z)\right)^{\delta}>1-\frac{4 \delta(M-1)}{n} \log 2, \quad\left(z \in U^{*}\right) .
$$

The bound in (35) is the best possible.

Letting $\delta=1, M=1+n / 8 \log 2$, and $t=0$ in Corollary 6 , we have the following. 
Corollary 7. Let $f^{\prime}(z) \neq 0$ for $z \in U^{*}$ and suppose that

$$
\Re\left\{z f(z)^{\prime}\left(1+\frac{z f^{\prime \prime}(z)}{2 f^{\prime}(z)}\right)\right\}<1+\frac{n}{8 \log 2} .
$$

Then

$$
\mathfrak{R}\left\{z f(z)^{\prime}\right\}>0, \quad\left(z \in U^{*}\right) .
$$

The result is sharp.

Theorem 8. Let $\xi>0, z\left(L_{a}^{t}(\alpha+1, \beta) f(z)\right)^{\prime} / L_{a}^{t}(\alpha, \beta) f(z) \neq 0$ for $z \in U^{*}$ and suppose that

$$
\begin{aligned}
\mathfrak{R}\{ & 1+\left(\frac{L_{a}^{t}(\alpha, \beta) f(z)}{L_{a}^{t}(\alpha+1, \beta) f(z)}\right)^{\xi} \\
& \times\left(\frac{(\alpha+1) L_{a}^{t}(\alpha+2, \beta) f(z)}{L_{a}^{t}(\alpha+1, \beta) f(z)}\right) \\
& \left.-\alpha\left(\frac{L_{a}^{t}(\alpha, \beta) f(z)}{L_{a}^{t}(\alpha+1, \beta) f(z)}\right)^{\xi}-1\right\}<M,
\end{aligned}
$$

where

$$
1<M \leq 1+\frac{n}{2 \xi \log 2}
$$

Then

$$
\begin{aligned}
& \Re\left(\frac{L_{a}^{t}(\alpha, \beta) f(z)}{L_{a}^{t}(\alpha+1, \beta) f(z)}\right)^{\xi} \\
& \quad>1-\frac{2 \xi(M-1)}{n} \log 2 \quad\left(z \in U^{*}\right) .
\end{aligned}
$$

The bound in (40) is the best possible.

Proof. Define the function $q(z)$ by

$$
q(z)=\left(\frac{L_{a}^{t}(\alpha+1, \beta) f(z)}{L_{a}^{t}(\alpha, \beta) f(z)}\right)^{\xi}
$$

Then, clearly $q(z)=1+q_{n} z^{n}+q_{n+1} z^{n+1}+\cdots$ analytic function in $U^{*}$ with $q(z) \neq 0$ for $z \in U^{*}$. Also by a simple computation and by making use of the familiar identity (11), we find from (41) that

$$
\begin{aligned}
1+\frac{1}{\xi} \frac{z q^{\prime}(z)}{q^{2}(z)}=1 & +\left(\frac{L_{a}^{t}(\alpha, \beta) f(z)}{L_{a}^{t}(\alpha+1, \beta) f(z)}\right)^{\xi} \\
& \times\left(\frac{(\alpha+1) L_{a}^{t}(\alpha+2, \beta) f(z)}{L_{a}^{t}(\alpha+1, \beta) f(z)}\right. \\
& \left.-\alpha\left(\frac{L_{a}^{t}(\alpha, \beta) f(z)}{L_{a}^{t}(\alpha+1, \beta) f(z)}\right)^{\xi}-1\right) .
\end{aligned}
$$

Applying Lemma 1, with $a=1 / \xi$, we get the required result.
Letting $\alpha=\beta=1$ in Theorem 8 , we have the following.

Corollary 9. Let $\xi>0, z\left(G_{t, a}(z)\right)^{\prime} / G_{t, a}(z) \neq 0$ for $z \in U^{*}$ and suppose that

$$
\begin{aligned}
\mathfrak{R}\{1 & +\left(\frac{G_{t, a}(z)}{z\left(G_{t, a}(z)\right)^{\prime}}\right)^{\xi} \\
& \left.\times\left(1+\frac{z\left(G_{t, a}(z)\right)^{\prime \prime}}{\left(G_{t, a}(z)\right)}-\left(\frac{G_{t, a}(z)}{z\left(G_{t, a}(z)\right)^{\prime}}\right)^{\xi}\right)\right\}<M,
\end{aligned}
$$

where

$$
1<M \leq 1+\frac{n}{2 \xi \log 2}
$$

Then

$$
\Re\left(\frac{G_{t, a}(z)}{z\left(G_{t, a}(z)\right)^{\prime}}\right)^{\xi}>1-\frac{2 \xi(M-1)}{n} \log 2, \quad(z \in U) .
$$

The bound in (45) is the best possible.

Letting $\xi=1, M=1+n / 2 \log 2$, and $t=0$ in Corollary 9, we have the following.

Corollary 10. Let $z f^{\prime}(z) / f(z) \neq 0$ for $z \in U^{*}$ and suppose that

$$
\begin{aligned}
& \mathfrak{R}\left\{1+\left(\frac{f(z)}{z f^{\prime}(z)}\right)^{\xi}\left(1+\frac{z f^{\prime \prime}(z)}{f^{\prime}(z)}-\left(\frac{f(z)}{z f^{\prime}(z)}\right)^{\xi}\right)\right\} \\
& <1+\frac{n}{2 \log 2} .
\end{aligned}
$$

Then

$$
\Re\left(\frac{f(z)}{z f^{\prime}(z)}\right)>0, \quad\left(z \in U^{*}\right) .
$$

The result is sharp.

\section{Conflict of Interests}

The authors declare that they have no competing interests.

\section{Authors' Contribution}

Both authors read and approved the final paper.

\section{Acknowledgment}

The work here is supported by a special grant: DIP-2013-1.

\section{References}

[1] S. G. Krantz, "Meromorphic functions and singularities at infinity," in Handbook of Complex Variables, pp. 63-68, Birkhäauser, Boston, Mass, USA, 1999. 
[2] R. K. Pandey, Applied Complex Analysis, Discovery Publishing House, Grand Rapids, Mich, USA, 2008.

[3] H. M. Srivastava and A. A. Attiya, "An integral operator associated with the Hurwitz-Lerch Zeta function and differential subordination," Integral Transforms and Special Functions, vol. 18, no. 3, pp. 207-216, 2007.

[4] H. M. Srivastava and J. Choi, Series Associated with the Zeta and Related Functions, Kluwer Academic, Boston, Mass, USA, 2001.

[5] H. M. Srivastava, D. Jankov, T. K. Pogány, and R. K. Saxena, "Two-sided inequalities for the extended Hurwitz-Lerch Zeta function," Computers and Mathematics with Applications, vol. 62, no. 1, pp. 516-522, 2011.

[6] H. M. Srivastava, R. K. Saxena, T. K. Pogany, and R. Saxena, "Integral transforms and special functions," Applied Mathematics and Computation, vol. 22, no. 7, pp. 487-506, 2011.

[7] J. Dziok and H. M. Srivastava, "Certain subclasses of analytic functions associated with the generalized hypergeometric function," Integral Transforms and Special Functions, vol. 14, no. 1, pp. 7-18, 2003.

[8] F. Ghanim and M. Darus, "A new class of meromorphically analytic functions with applications to the generalized hypergeometric functions," Abstract and Applied Analysis, vol. 2011, Article ID 159405, 10 pages, 2011.

[9] F. Ghanim and M. Darus, "Some properties of certain subclass of meromorphically multivalent functions defined by linear operator," Journal of Mathematics and Statistics, vol. 6, no. 1, pp. 34-41, 2010.

[10] F. Ghanim and M. Darus, "Some properties on a certain class of meromorphic functions related to Cho-Kwon-Srivastava operator," Asian-European Journal of Mathematics, vol. 5, no. 4, Article ID 1250052, pp. 1-9, 2012.

[11] J.-L. Liu and H. M. Srivastava, "Certain properties of the DziokSrivastava operator," Applied Mathematics and Computation, vol. 159, no. 2, pp. 485-493, 2004.

[12] J.-L. Liu and H. M. Srivastava, "Classes of meromorphically multivalent functions associated with the generalized hypergeometric function," Mathematical and Computer Modelling, vol. 39, no. 1, pp. 21-34, 2004.

[13] D. Yang, "Some criteria for multivalent starlikeness," Southeast Asian Bulletin of Mathematics, vol. 24, no. 3, pp. 491-497, 2000. 


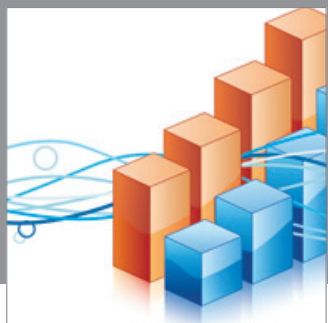

Advances in

Operations Research

mansans

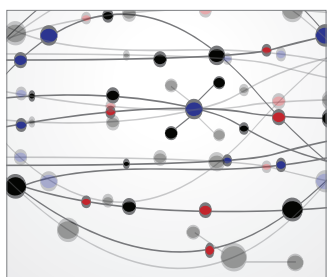

The Scientific World Journal
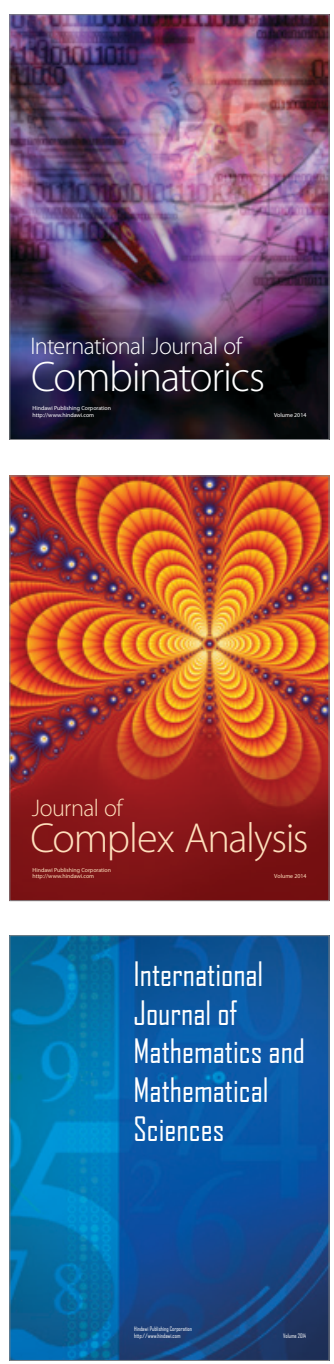
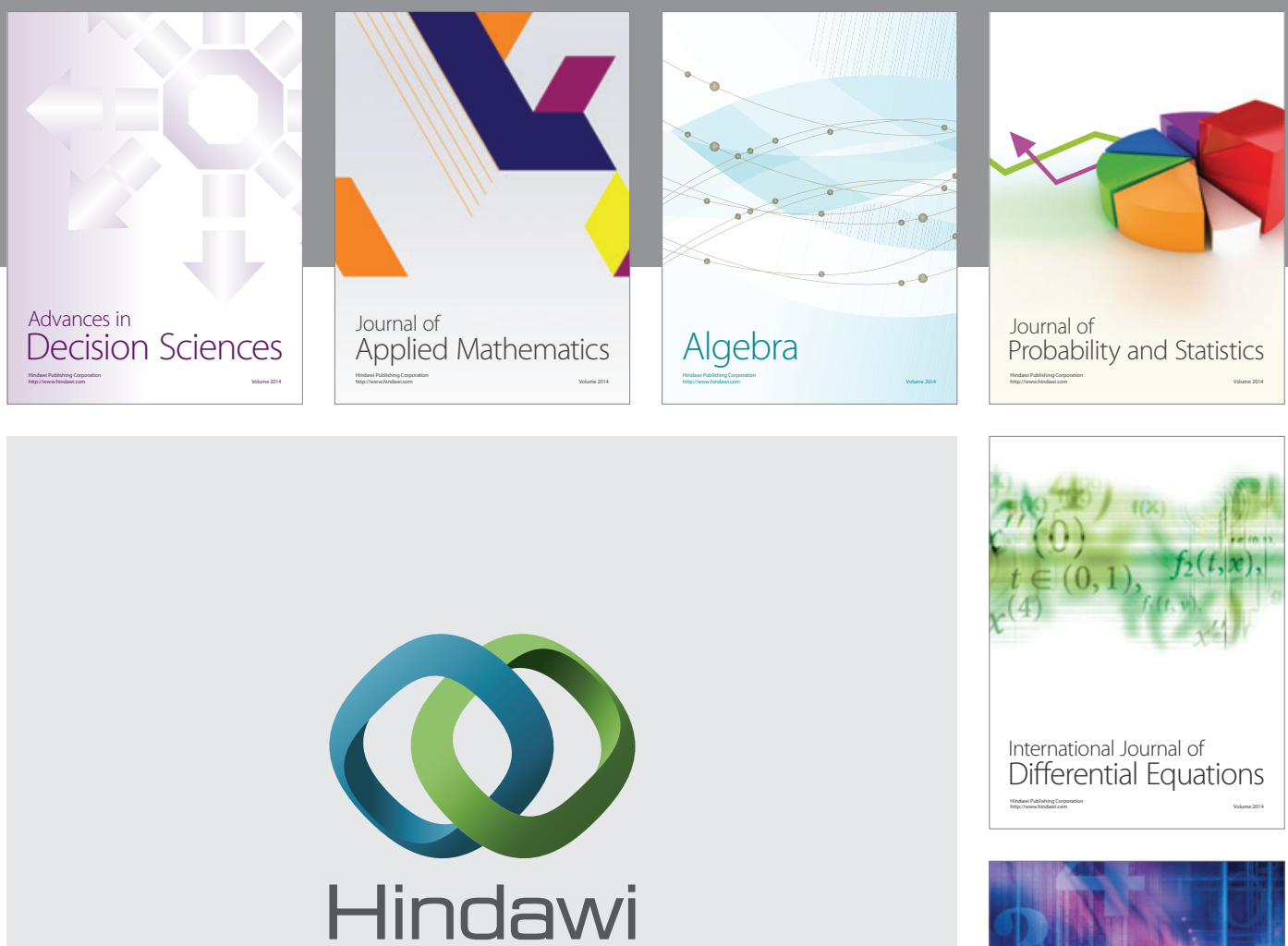

Submit your manuscripts at http://www.hindawi.com
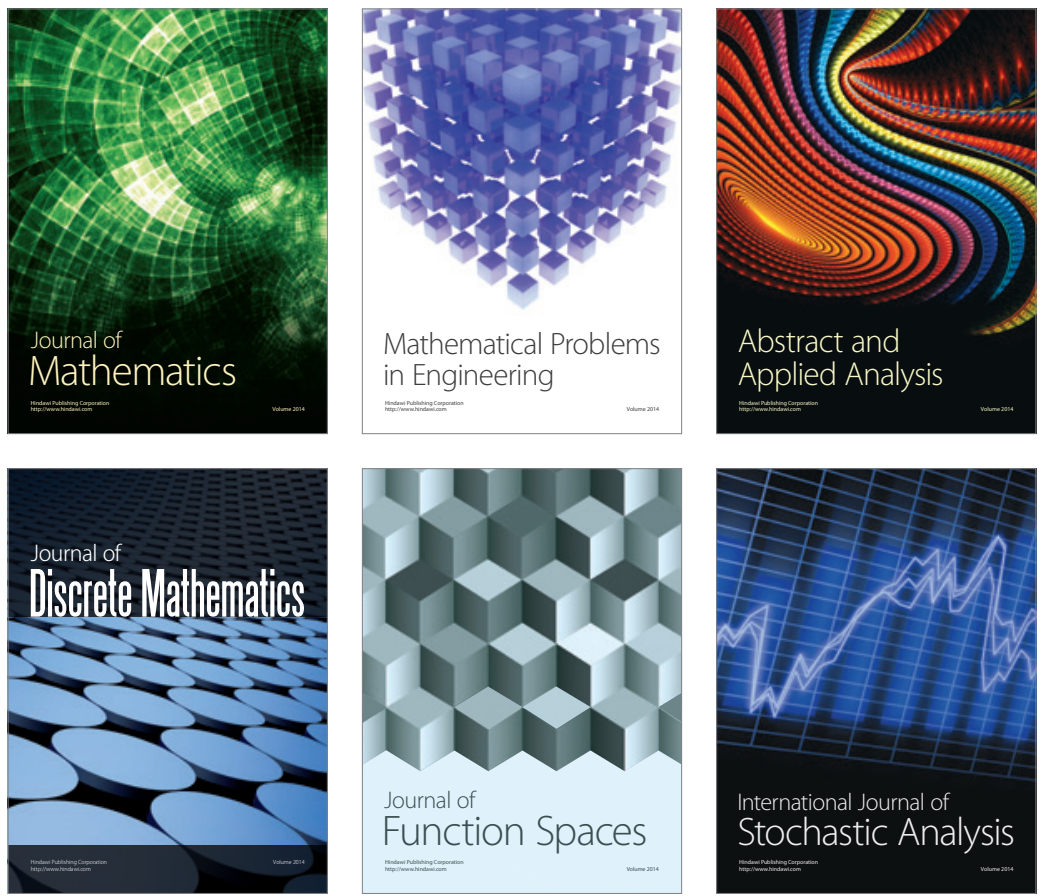

Journal of

Function Spaces

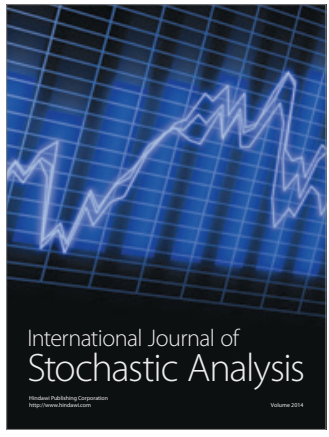

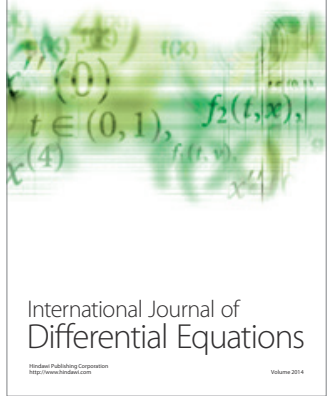
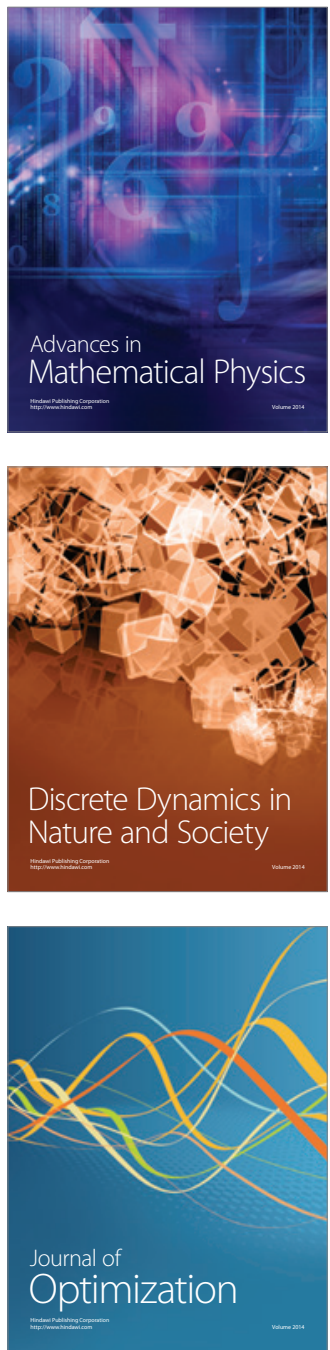